\title{
Diagnostic Accuracy of a Point-of-Care Test for Celiac Disease Antibody Screening among Infertile Patients
}

\author{
Louise Grode $^{\mathrm{a}}$ Thomas Møller Jensen ${ }^{\mathrm{a}}$ Tina Parkner ${ }^{\mathrm{b}}$ Inge Errebo Agerholm ${ }^{\mathrm{c}}$ \\ Peter Humaidan ${ }^{d, e}$ Bodil Hammer Bech ${ }^{f}$ Cecilia Ramlau-Hansen ${ }^{f}$ \\ ${ }^{a}$ Department of Medicine, Horsens Regional Hospital, Horsens, Denmark; ${ }^{b}$ Department of Clinical Biochemistry, \\ Arhus University Hospital, Aarhus, Denmark; ' Origio, Måløv, Denmark; ${ }^{d}$ Fertility Clinic, Skive Regional Hospital, \\ Skive, Denmark; ${ }^{\mathrm{e}}$ Faculty of Health, Aarhus University, Aarhus, Denmark; ${ }^{\mathrm{f}}$ Department of Public Health, Aarhus \\ University, Aarhus, Denmark
}

\section{Keywords}

Early detection - Screening $\cdot$ Celiac disease $\cdot$ Rapid test .

Gluten intolerance

\begin{abstract}
Background: Screening for celiac disease among infertile patients has been suggested. Several rapid point-of-care (POC) tests aimed at detecting celiac disease antibodies have been developed. It has been suggested that these POC tests can be implemented as a replacement for standard laboratory tests. Objective: To evaluate the diagnostic accuracy of a POC test (Simtomax ${ }^{\circledR}$ ) that detects celiac disease antibodies compared with standard laboratory tests when screening for celiac disease among patients referred for fertility treatment in 2 Danish fertility clinics. Methods: Serum samples were analyzed for lgA anti-tissue transglutaminase (TGA) as the reference standard test with a cutoff of $\geq 7 \mathrm{kU} / \mathrm{L}$ and by the index POC test based on IgA and IgG antibodies against deamidated gliadin peptides (DGP). In IgA deficien$c y$, the reference standard test was IgG DGP with a cutoff of $\geq 7 \mathrm{kU} / \mathrm{L}$. Participants answered a questionnaire on gluten
\end{abstract}

intake, symptoms, and risk factors. Diagnostic confirmation was made by duodenal biopsies. IgA TGA/lgG DGP were used as the reference standard to calculate positive and negative predictive values. Results: A total of 622 men and women (51.6\%) were enrolled during 2015. The reference standard IgA TGA/lgG DGP was positive in 7 participants $(1.1 \%$ [95\% $\mathrm{Cl} 0.5-2.3])$ and the $\mathrm{POC}$ test was positive in 84 participants (13.5\% [95\% Cl 10.9-16.4]), 3 of whom also had positive reference standard tests. This yields a sensitivity of the index POC test of $42.9 \%(95 \% \mathrm{Cl} 9.9-81.6)$ and a specificity of 86.8\% (95\% Cl 83.9-89.4). Positive and negative predictive values were $3.57 \%(95 \% \mathrm{Cl} 0.7-10.1)$ and $99.3 \%$ (95\% Cl 98.199.8). Conclusion: The sensitivity of the POC test was low; however, the specificity was moderately good. The POC test had a high negative predictive value in this low prevalent population but missed 1 patient with biopsy-confirmed celiac disease. However, because of many false-positive tests, it cannot be recommended as replacement for standard laboratory tests but rather as a triage test to decide if the standard serology tests should be performed.

(c) 2019 S. Karger AG, Base

\section{KARGER}

(c) 2019 S. Karger AG, Basel

E-Mail karger@karger.com

www.karger.com/iid
Louise Grode

Department of Medicine, Horsens Regional Hospital

Sundvej 30

DK-8700 Horsens (Denmark)

E-Mail louise.grode@ horsens.rm.dk 


\section{Introduction}

Celiac disease is an autoimmune disease triggered by the ingestion of gluten proteins from wheat, barley, and rye. Gluten proteins induce T-cell-mediated inflammation in the small bowel with loss of villous structure and an autoimmune response to self-proteins, mainly tissue transglutaminase [1]. The classical symptoms of celiac disease are gastrointestinal symptoms such as abdominal pain and diarrhea. However, since the advent of serological testing, most celiac disease cases have been found with no, minimal, or nonclassical symptoms, and, therefore, the diagnosis of celiac disease is often missed or delayed for years [2]. Objective signs and symptoms seem to improve when patients commence on a gluten-free diet, including elimination of circulating tissue transglutaminase antibodies (TGA) and remission of villous structure [3]. Celiac disease affects up to $1 \%$ of the general population in Europe and the USA with some regional differences $[4,5]$, but only $10-15 \%$ are believed to be diagnosed [6-8]. In Denmark, $0.18 \%$ of the population had a diagnosis of celiac disease in 2016 [9], but a screening study found a prevalence of $0.48 \%$ in a sample of the general Danish population [10], indicating that unrecognized celiac disease is frequent. Early diagnosis and treatment are warranted, and it has been discussed whether celiac disease screening of risk groups can be justified to increase the diagnostic rate [11]. Celiac disease has been linked to infertility and adverse pregnancy outcomes [12-15]. Therefore, patients with fertility problems could be seen as a risk group, and, as a consequence, routine screening for celiac disease in fertility treatment programs has been suggested $[16,17]$.

The initial diagnosis of celiac disease is made by serological testing [18-20], but in adults the diagnosis should be confirmed by histological findings in biopsies from the small bowel. The most sensitive serological tests for celiac disease are IgA tissue TGA (IgA TGA) or IgA endomysial antibody (IgA EMA). IgA TGA is the most commonly preferred test because it is less costly. It is recommended also to test for total IgA, since an IgA TGA/EMA test will be negative if the patient suffers from IgA deficiency. If IgA deficiency is present, it is the standard to test for IgG deamidated gliadin peptide (DGP) [8]. Despite excellent serological celiac disease testing with high accuracy, several rapid point-of-care (POC) tests detecting celiac disease antibodies have been developed. They have some advantages in terms of the swift delivery of results without the need for special- ized equipment leaving an opportunity of testing outside the hospital environment [21]. These advantages could lead to increased testing and a higher diagnostic rate. The diagnostic accuracy of these POC tests in detecting patients with celiac disease is highly relevant, and it is important to evaluate the performance of these tests and clinical use in relevant settings. The Simtomax ${ }^{\circledR}$ POC test detects celiac disease antibodies and IgA deficiency, and it is a qualitative immunochromatographic POC test to be visually read. The intended use is as a first-line screening test for celiac disease antibodies in nonspecialist settings. Previously, Simtomax ${ }^{\circledR}$ has primarily been tested among patients representing high-prevalence populations as in gastroenterological specialist settings $[20,22-28]$. The accuracy of Simtomax ${ }^{\circledR}$ has not yet been tested in a fertility treatment program with an expected lower prevalence of celiac disease.

The aim of this study was to evaluate the performance of a serologically based POC test (Simtomax ${ }^{\circledR}$ ) aimed at detecting celiac disease antibodies in a population of men and women referred for fertility treatment in 2 Danish public fertility clinics.

\section{Materials and Methods}

\section{Study Population}

Heterosexual couples referred for fertility treatment, having a Danish address, and being able to read and understand Danish were considered potentially eligible. By their medical records, using the ICD-8 code 269.00 and ICD-10 code K90.0, we identified potential participants who already had a diagnosis of celiac disease and excluded them with their partner from the study.

\section{Questionnaires on Symptoms and Predisposing Factors}

To detect possible risk factors and gastrointestinal symptoms, all enrolled participants answered the Gastrointestinal Symptom Rating Scale (GSRS) [29] and questions about family history of celiac disease, other comorbidities, autoimmune diseases, and fertility history. The questionnaire also included the question "Do you avoid gluten intake in your daily diet?" to allow identification of participants where celiac disease antibody screening could potentially be invalid. The GSRS questionnaire is a validated, selfadministered questionnaire that includes 15 questions, originally designed to detect irritable bowel syndrome and peptic ulcer disease, but it is also used to detect symptoms in celiac disease [29]. The GSRS assesses severity of gastrointestinal symptoms using a 7-point Likert scale in 5 domains: indigestion, diarrhea, constipation, abdominal pain, and reflux. The severity of symptoms reported in the GSRS increases with increasing score, and an average score is calculated within each domain. Participants were asked to answer the study questionnaire at the fertility clinic on the same day as blood samples were drawn. 


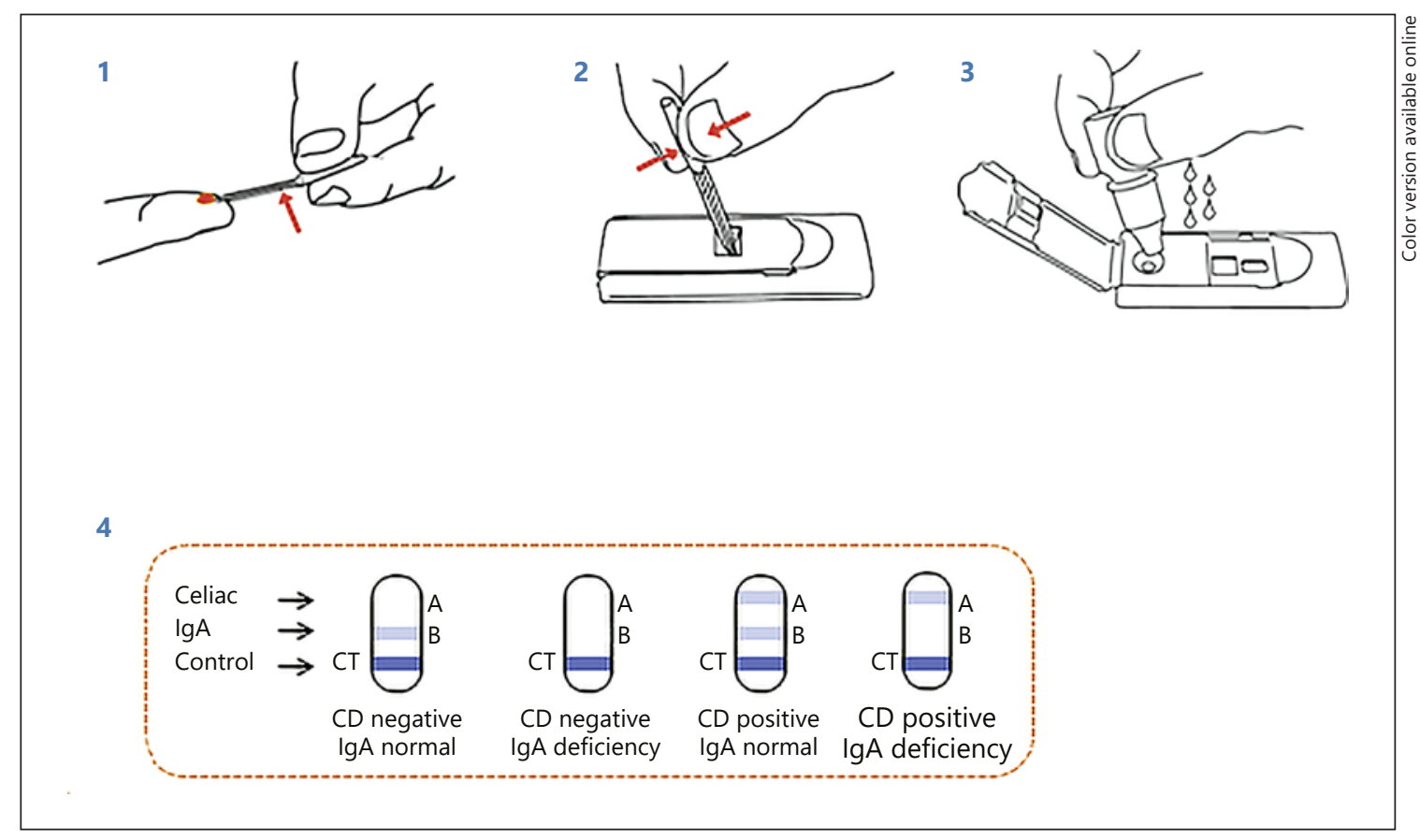

Fig. 1. Simtomax ${ }^{\circledR}$ POC test device and interpretation of test results as visualized by the manufacturer. CD, celiac disease.

\section{Data Collection}

After the patient had given informed consent, whole-blood samples (3-6 mL) were drawn. Two samples were used for the analysis of the reference standard laboratory tests performed in the hospital laboratory, and 1 blood sample was used for the index POC test. The reference standard tests and the index POC tests were analyzed in 2 different settings, and when performing the index POC tests, the results of the reference standard tests were unknown.

Prior to the initiation of the study, representatives from the Simtomax ${ }^{\circledR}$ manufactoring company (Augurix and Tillots Pharma) conducted training sessions for all study associates and the biomedical laboratory scientists at the 2 fertility clinics.

\section{Reference Standard Test}

Using a fluorescence enzyme immunoassay (ImmunoCAP 250; Phadia, Uppsala, Sweden), serum was analyzed for IgA TGA and total IgA. Because IgA deficiency is associated with a higher risk of celiac disease and IgA TGA is invalid in IgA deficiency, all samples with a total IgA level $\leq 0.8 \mathrm{~g} / \mathrm{L}$ were tested for IgG DGP as another marker for celiac disease. The cutoff values for a positive test indicating celiac disease were IgA TGA $\geq 7 \mathrm{kU} / \mathrm{L}$ or IgG DGP $\geq 7 \mathrm{kU} / \mathrm{L}$ in line with the hospital standard laboratory guidelines at the time of study conduction. The range between 7 and $10 \mathrm{kU} / \mathrm{L}$ is classified as a "gray area" in diagnosing celiac disease for both IgA TGA and IgG DGP.

\section{Index POC Test}

The Simtomax ${ }^{\circledR}$ POC test is manufactured by Augurix Diagnostics (Switzerland) and detects IgA and IgG DGP and total IgA in either $25 \mu \mathrm{L}$ of capillary whole blood from a fingertip or $20 \mu \mathrm{L}$ serum or plasma (EDTA or heparinized). In the present study, 20 $\mu \mathrm{L}$ of serum were used. The exact cutoff values could not be specified by the manufacturing company. The test results can be visually read after $10 \mathrm{~min}$. Test results have 6 different outcomes for interpretation. Two test outcomes indicate an invalid test, and the test should be repeated. Four of the outcomes indicate different result combinations of celiac disease and IgA deficiency (Fig. 1).

\section{Confirmation of Diagnosis by Histology}

Even though increasing serological antibody levels (TGA, DGP, and EMA) correlate with increasing diagnostic certainty set by the histological changes in the small bowel, a cutoff value for absolute diagnostic certainty for the antibodies has still not been established. Therefore, in this study, biopsies from the small bowel (4-5 duodenum and duodenal bulb biopsies) were taken during a gastroscopy to confirm the celiac disease diagnosis in case of positive standard serological tests. Biopsy material was sent to pathology for histological classification. The histology was classified using the 4-stage, modified "Marsh-Oberhuber criteria" determining the classical histological changes seen in celiac disease [30, 31].

For ethical reasons, we used a partial biopsy verification strategy if the reference standard tests (IgA TGA or IgG DPG) were negative, but the index POC test indicated celiac disease. The participant required at least 1 of the following criteria to be referred to a gastroscopy with small-bowel biopsies: a minimum score of 4 (indicating some problems) in at least 1 of the GSRS domains: diarrhea, indigestion, or abdominal pain and/or a diagnosis of diabetes type 1, thyroiditis, Graves/Basedow disease, Sjögren disease, a first-degree relative with celiac disease, or depression with medical treatment. Participants who marked in the questionnaire that they suffered from depression and the index POC test indicated 
Table 1. Baseline characteristics of the participants

\begin{tabular}{lc}
\hline Female gender, $n$ & $321(51.6 \%)$ \\
Median age (min-max), years & $30.4(21.6-40.9)$ \\
$\quad$ Women & $32.0(21.7-55.0)$ \\
Men & \\
Median BMI (min-max), kg/m² & $26.0(18.4-42.1)$ \\
$\quad$ Women & $24.8(17.0-40.2)$ \\
Men & $45(7.3 \%)$ \\
Avoiding gluten in diet, $n$ & $8(1.3 \%)$ \\
Data missing & $11(1.8 \%)$ \\
Risk factors, $n$ & $8(1.3 \%)$ \\
First-degree relatives with CD & $13(2.3 \%)$ \\
Diabetes type 1 & $6(1.0 \%)$ \\
Thyroiditis & 0 \\
Graves disease & $63(10.3 \%)$ \\
Sjögren disease & \\
Depression & $16(2.7 \%)$ \\
Gastrointestinal symptoms (GSRS $\geq 4), n$ & $n$ \\
Diarrhea & $24(4 \%)$ \\
Indigestion & $5(0.8 \%)$ \\
Abdominal pain & $13(2.1 \%)$ \\
Reflux & $14(2.3 \%)$ \\
Constipation & \\
\hline
\end{tabular}

Table 2. Index POC test results compared with IgA TGA/IgG DGP as reference standard

\begin{tabular}{lccc}
\hline & $\begin{array}{l}\text { POC test } \\
\text { positive }\end{array}$ & $\begin{array}{l}\text { POC test } \\
\text { negative }\end{array}$ & Total \\
\hline Standard test positive & 3 & 4 & 7 \\
Standard test negative & 81 & 534 & 615 \\
\hline Total & 84 & 538 & 622 \\
\hline
\end{tabular}

celiac disease were contacted to clarify if they received current antidepressants. If fulfilling at least 1 of these criteria, the participant was classified as at "high risk of celiac disease."

If IgA TGA or IgG DGP exceeded the cutoff values or the index POC test indicated celiac disease and the participant was classified as at "high risk of celiac disease," the participant was contacted by the responsible gastroenterologist and informed of the results and invited to a clinical examination (here determining other clinical symptoms) and a gastroscopy at the hospital.

A Marsh stage 0 with IgA TGA or IgG DGP exceeding the cutoff values may indicate latent celiac disease, but only patients with Marsh stage 1 or 2 and with clinical symptoms of celiac disease and patients with Marsh stage $3(\mathrm{a}-\mathrm{c})$ classified histology were considered as having biopsy-confirmed celiac disease.

\section{Statistical Analyses}

Sensitivity, specificity, and positive (PPV) and negative predictive values (PNV) for the index POC test were calculated with IgA TGA/IgG DGP as reference standard. Positive $(\mathrm{LH}+)$ and negative likelihood ratios (LH-) and test accuracy were also calculated from the sensitivity and specificity. The LH are less dependent on the prevalence of the disease in the study sample and can be used to calculate posttest probability adapting different pretest probabilities for disease (prevalence) from different contexts. All values are given with 95\% confidence intervals (95\% CI). Analyses were performed using Stata version 14 (Stata Corporation, College Station, TX, USA).

\section{Results}

A total of 643 men and women were consecutively enrolled in the study during the period from January to December 2015. The baseline characteristics of all participants are described in Table 1 . The reference standard and the index POC tests were performed in 622 participants (96.7\%). In 21 participants, either blood samples were missing or one of the tests had not been performed. The questionnaire was answered by 611 (98.2\%) of the 622 participants screened.

Of these 622 participants, 7 patients had IgA TGA/IgG DGP exceeding the cutoff values, yielding a prevalence of reference standard celiac disease antibody-positive tests of $1.1 \%$ (95\% CI 0.5-2.3). The index POC test was indicating celiac disease in 84 participants (13.5\% [95\% CI 10.616.4]). Of the 7 participants with a positive standard test, 3 also had a positive index POC test indicating celiac disease, and 4 had a negative index POC test (Tables 2, 3). All 7 participants with a standard positive test underwent gastroscopy, and 4 participants also had histological changes corresponding to celiac disease, and 3 of these also had an index POC test indicating celiac disease. Only 1 of the 7 standard test-positive cases had any of the predefined risk factors. Of the 81 cases where the standard test was normal, but the index POC test indicated celiac disease, 14 cases (17.3\% [95\% CI 9.7-27.3]) were classified as at "high risk of celiac disease" with a GSRS score $\geq 4$ or other risk factors and were offered gastroscopy (Fig. 2). Of the 14 "high-risk celiac disease" cases, 4 (4.9\% [95\% CI 1.4-12.2]) had depression with medical treatment and $10(12.3 \%$ [95\% CI 6.1-21.5]) had one of the other risk factors. Among participants with normal standard and index POC tests $(n=534), 58$ participants $(10.9 \%$ [95\% CI 8.413.8]) could be classified as "high-risk celiac disease" if not including depression. Depression with medical treatment was not calculated in this group since their POC tests were normal, and, therefore, they were not contacted to clarify if they received antidepressants.

Of the standard test-negative cases having an index POC test indicating celiac disease and classified as "highrisk celiac disease" $(n=14)$, none were found with histo- 


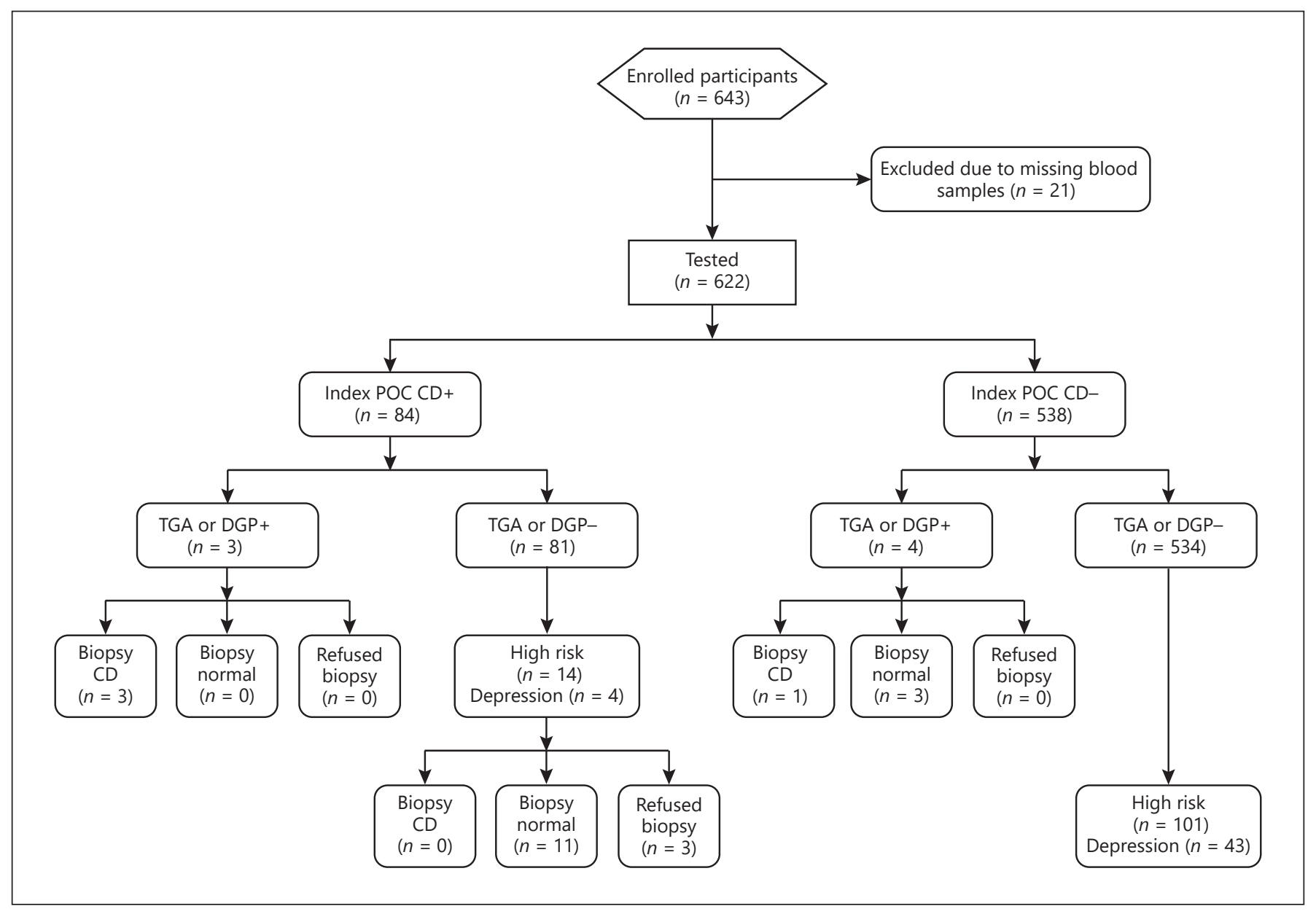

Fig. 2. Flow diagram of participants. $C D$, celiac disease.

Table 3. Index POC test results compared with histological classification in 21 biopsied participants

\begin{tabular}{lclc}
\hline & $\begin{array}{l}\text { POC test } \\
\text { positive }\end{array}$ & $\begin{array}{l}\text { POC test } \\
\text { negative }\end{array}$ & Total \\
\hline Marsh 1-3 (a, b, c) & 3 & 1 & 4 \\
Marsh 0 & 14 & 3 & 17 \\
\hline Total & 17 & 4 & 21 \\
\hline
\end{tabular}

logical changes corresponding to celiac disease ( 3 declined gastroscopy and further clinical examination).

Total IgA levels $\leq 0.8 \mathrm{~g} / \mathrm{L}$ were present in 10 participants (1.6\% [95\% CI 0.8-2.9]) where IgG DGP levels were measured. The index POC test indicated IgA deficiency in 21 participants, whereof 1 had total $\operatorname{IgA} \leq 0.8 \mathrm{~g} / \mathrm{L}$. Of 45 participants saying that they were avoiding gluten, the standard reference tests were celiac disease antibody positive in 1 participant, and the index POC test indicated celiac disease in 8 participants.

\section{Test Accuracy}

The index POC test had a sensitivity of $42.9 \%$ (95\% CI 9.9-81.6) and a specificity of $86.8 \%$ (95\% CI 83.9-89.4) using the IgA TGA/IgG DGP as reference standard with the cutoff values chosen prior to study start. In this lowprevalence population, the PPV was 3.57\% (95\% CI 0.710.1 ) and the NPV 99.3\% (95\% CI 98.1-99.8). The LH+ was 3.25 (95\% CI 1.35-7.84) and the LH- 0.66 (95\% CI $0.35-1.25)$. The overall probability that a participant would correctly be classified by the index POC test (test accuracy) was $86.3 \%$ (95\% CI 83.4-88.9) (Table 4). If the index POC test was compared with IgA TGA/IgG DGPseropositive biopsies confirming celiac disease $(n=4)$, the sensitivity changed to $75 \%$ (95\% CI 19.4-99.4). 
Table 4. Index POC test compared with IgA TGA/IgG DGP as reference standard

\begin{tabular}{|c|c|c|}
\hline \multicolumn{2}{|l|}{ Measure } & $95 \%$ CI \\
\hline \multicolumn{3}{|c|}{ Prevalence of standard test exceeding } \\
\hline cutoff & $1.1 \%$ & $0.45-2.3$ \\
\hline Sensitivity & $42.9 \%$ & $9.9-81.6$ \\
\hline Specificity & $86.8 \%$ & $83.9-89.4$ \\
\hline Positive predictive value & $3.57 \%$ & $0.7-10.1$ \\
\hline Negative predictive value & $99.3 \%$ & $98.1-99.8$ \\
\hline Likelihood ratio positive & 3.25 & $1.35-7.84$ \\
\hline Likelihood ratio negative & 0.66 & $0.35-1.25$ \\
\hline Test accuracy & $86.3 \%$ & $83.4-88.9$ \\
\hline
\end{tabular}

\section{Discussion}

It is important to test a new diagnostic test in populations and settings similar to those where it is intended to be used. According to the manufacturer, the intended use of the Simtomax ${ }^{\circledR}$ POC test is as first-line screening of patients suspected of celiac disease, e.g., in primary care or other celiac disease nonspecialist areas. Fertility clinics could be defined as a celiac disease nonspecialist area, and infertility may be a nonclassical symptom of celiac disease leading to suspicion of undetected celiac disease.

With a sensitivity of $42.9 \%$ (95\% CI 9.9-81.6), the POC test did not perform well in detecting those who were true positive for celiac disease antibodies according to the reference standard tests; however, the specificity was $86.8 \%$, which means the index POC test performed moderately well in identifying those who were true negative according to the reference standard test. A low sensitivity makes a test unsuitable for screening for severe or fatal disease since the test will miss too many truly diseased patients. Although it is not known if asymptomatic celiac patients are at risk of severe complications [32] and those not detected by the index POC test had low antibody levels within the "gray zone," biopsies confirmed celiac disease in 1 asymptomatic patient with an IgA TGA of $7 \mathrm{kU} / \mathrm{L}$. Changing the cutoff levels for the standard tests will impact the POC test performance. A low cutoff is set to minimize the risk of overlooking disease in clinical practice. The cutoffs for the reference standard tests in this study were set according to the Danish guidelines for laboratory work used at the hospital laboratory. The "gray zones" up to $10 \mathrm{kU} / \mathrm{L}$ for the standard tests are defined as diagnostically inconclusive zones compared with histological classification as the reference standard for diagnosing celiac disease. Changing the cutoff of IgA TGA to $>10 \mathrm{kU} / \mathrm{L}$ would increase the sen- sitivity of the Simtomax ${ }^{\circledR}$ POC test since it was not able to capture low levels of celiac disease antibodies.

The main interest in practice is the ability of the diagnostic tests to predict whether celiac disease antibodies are present or not, i.e., the PPV and NPV, and how the test can alter the posttest probability (LH ratio). The PPV and NPV are dependent on the prevalence of the disease in the population in which the test is used, and, therefore, the test can have different predictive values in different settings, e.g., a nonspecialist versus a specialist setting. Previously reported PPV and NPV for the Simtomax ${ }^{\circledR}$ POC test from pediatric or gastroenterological settings $[25,26]$ cannot be expected to be the same in a population of infertile patients with an expected lower prevalence of celiac disease. With an NPV of $99.3 \%$ (95\% CI 98.1-99.8), there is a very high certainty that a patient with a negative POC test in fact is celiac disease antibody negative if the pretest risk of being celiac disease antibody positive is as low as $1.1 \%$. The $\mathrm{LH}$ ratios can be useful in clinical practice to calculate the posttest probability in different pretest probabilities. With a pretest probability of $1.1 \%$ and a $\mathrm{LH}+$ of 3.25 , the posttest probability or "risk" for in fact being antibody positive given a positive index POC test changed from 1.1 to $4 \%$ [33].

The pretest probability of being celiac disease antibody positive (prevalence of positive standard tests in this population) was unknown at study start but expected to be $2.0 \%$ (the prevalence of biopsy-confirmed celiac disease was expected to be $1.75 \%$ ), presumably resulting in 12 celiac disease antibody cases in our study population. The pretest probability was lower than expected with a prevalence of $1.1 \%$.

To investigate whether a negative standard test but a positive index POC test in patients with classical celiac disease symptoms or with known risk factors predicted celiac disease in biopsies, we invited these participants to have gastroscopy performed $(n=14)$. A total of 11 participants accepted gastroscopy, but none had histological changes indicating celiac disease. Interestingly, only 1 of the standard test-positive cases had any of the "high-risk celiac disease" factors, which means that if the index POC test solely was used in patients with classical symptoms or risk factors, only 1 of the 7 participants with a reference standard-positive test would be suspected and tested for celiac disease.

Neither the standard tests nor the index test is diagnostically conclusive regarding celiac disease, only histological changes in the small bowel are diagnostically conclusive. The tests are intended for initial screening of patients who should be referred to gastroscopy with biopsies. The index POC test indicated celiac disease in 84 participants, which implies that if the index POC test were 
used in clinical practice instead of the standard IgA TGA/ IgG DGP laboratory work, 84 patients would be referred to gastroscopy with biopsies, and 4 with standard IgA TGA/IgG DGP-positive tests would not be referred to gastroscopy. Undergoing a gastroscopy is distressing and not without risk of complications, and, moreover, it is costly. Therefore, in our opinion, the index POC test may be more suitable as a triage test to identify who should have the standard serology test performed.

The economical and clinical benefits of POC tests compared with standard laboratory tests in screening for celiac disease have only been sparsely studied. It is still unclear where exactly in the clinical pathway it would have its place. The question remains whether it is a replacement for the standard laboratory tests or a triage test to identify who should have the standard laboratory tests. Esteve et al. [34], who investigated the Simtomax ${ }^{\circledR}$ POC test, reported that a strategy with a positive POC test as a direct referral to gastroscopy was more cost effective than positive standard laboratory tests (IgA TGA) as a base for referral to gastroscopy. The savings were primarily found in fewer reconsultations; in other health care systems, these savings may not be relevant if reconsultations are for example handled by phone consultations. However, in their study, the Simtomax ${ }^{\circledR}$ POC test showed a sensitivity of $100 \%$ (95\% CI $40-100)$ and a specificity of $93 \%$ (95\% CI 89-95) in a population with a celiac disease prevalence of $1.14 \%$ [34]. The POC test performed better in the study by Esteve et al. [34], however, with overlapping 95\% CI regarding sensitivity compared with our results. The POC test is to be visually read, and some intra- and interreader variability must be expected, which were not assessed in the studies, and differences in the cutoff limits for the standard laboratory tests may also have influenced the results. With an NPV of $99.3 \%$, the Simtomax ${ }^{\circledR}$ POC test performed well in ruling out celiac disease antibodies; however, we cannot recommend it as replacement for the standard laboratory tests because of too many false-positive tests (13\%) leading to a gastroscopy referral.

\section{Strengths and Limitations of this Study}

The study used a design in which the study sample was defined as the base population in which the index POC test was intended to be used. The study was performed in a real-life routine clinical setting with a prospective data collection. The index POC test was handled and interpreted by the health care professionals to whom the test was intended and after training by the manufacturer. We suggest that this gives a realistic picture of how the index POC test would perform in an everyday clinical setting.
A significantly larger sample size would be required to increase statistical precision, as the prevalence of celiac disease antibody-positive cases was lower than expected. Finally, the Simtomax ${ }^{\circledR}$ POC test is a qualitative test to be visually read; however, the intra- and interrater agreement was not assessed, and, hence, disagreement in interpretation of the index POC tests was not assessed.

\section{Conclusion}

The POC test performed moderately well in identifying those with true-negative but poorly in identifying those with true-positive celiac disease antibody tests. The NPV was high in a population of infertile patients with a low prevalence of celiac disease antibody-positive patients. However, the index POC test had only moderate sensitivity with false-positive results in $13 \%$ of the patients, and, on this basis, a strategy of POC testing as replacement of the standard serology tests to detect candidates suitable for a gastroscopy cannot be recommended; in addition, it remains unclear where in the clinical pathway this POC test is beneficial.

\section{Acknowledgments}

We are thankful to all the health care professionals working in the fertility clinics and in the Department of Biochemistry for their assistance in including participants and processing laboratory tests in this study. Thanks go also to Tillots Pharma for supervision and training in how to use the Simtomax ${ }^{\circledR}$ POC test kits.

\section{Statement of Ethics}

The Ethics Committee of the Central Denmark Region approved the study (approval date: 27.11.2014 under J No. 1-10-72321-14). All participants gave written informed consent before inclusion. The study protocol conformed to the ethical guidelines of the 1975 Declaration of Helsinki. The study was also approved by the Danish Data Protection Agency (J No. 1-16-02-567-14).

\section{Disclosure Statement}

The authors have no conflict of interest to declare.

\section{Funding Sources}

This study was supported by grants from the Health Research Fund of Central Denmark (R38-A1465-B121) and an unconditional research grant by Tillotts Pharma AG, Switzerland. 


\section{Author Contributions}

Louise Grode: study design, acquisition, statistical analysis, interpretation of the data, and writing of the manuscript; Thomas Møller Jensen: study design, acquisition, interpretation of the data, and revision of the manuscript; Tina Parkner, Inge Errebo Agerholm, and Peter Humaidan: study design, acquisition, revision of the manuscript; Bodil Hammer Bech and Cecilia H. Ramlau-Hansen: Study design, interpretation of the data, revision of the manuscript. All authors read and approved the final article.

\section{References}

1 Sollid LM. Coeliac disease: dissecting a complex inflammatory disordert]. Nat Rev Immunol. 2002 Sep;2(9):647-55.

2 Fasano A, Catassi C. Clinical practice. Celiac disease. N Engl J Med. 2012 Dec;367(25): 2419-26.

3 Rubio-Tapia A, Rahim MW, See JA, Lahr BD, $\mathrm{Wu}$ TT, Murray JA. Mucosal recovery and mortality in adults with celiac disease after treatment with a gluten-free diet. Am J Gastroenterol. 2010 Jun;105(6):1412-20.

4 Singh P, Arora A, Strand TA, Leffler DA, Catassi C, Green PH, et al. Global Prevalence of Celiac Disease: Systematic Review and Metaanalysis. Clin Gastroenterol Hepatol. 2018 Jun;16(6):823-836.e2.

5 Catassi C, Gatti S, Lionetti E. World perspective and celiac disease epidemiology. Dig Dis. 2015;33(2):141-6.

6 Rubio-Tapia A, Ludvigsson JF, Brantner TL, Murray JA, Everhart JE. The prevalence of celiac disease in the United States. Am J Gastroenterol. 2012 Oct;107(10):1538-44.

7 Weile I, Grodzinsky E, Skogh T, Jordal R, Cavell B, Krasilnikoff PA. High prevalence rates of adult silent coeliac disease, as seen in Sweden, must be expected in Denmark. APMIS. 2001 Nov; 109(11):745-50.

8 NICE. Coeliac disease: recognition, assessment and management. NICE guideline [NG86]. Available from: www.nice.org.uk/ guidance/ng86.

9 Grode L, Bech BH, Jensen TM, Humaidan P, Agerholm IE, Plana-Ripoll O, et al. Prevalence, incidence, and autoimmune comorbidities of celiac disease: a nation-wide, population-based study in Denmark from 1977 to 2016. Eur J Gastroenterol Hepatol. 2018 Jan; 30(1):83-91.

10 Horwitz A, Skaaby T, Kårhus LL, Schwarz P, Jørgensen T, Rumessen JJ, et al. Screening for celiac disease in Danish adults. Scand J Gastroenterol. 2015 Jul;50(7):824-31.

11 Viljamaa M, Collin P, Huhtala H, Sievänen H, Mäki M, Kaukinen K. Is coeliac disease screening in risk groups justified? A fourteenyear follow-up with special focus on compliance and quality of life. Aliment Pharmacol Ther. 2005 Aug;22(4):317-24.

12 Grode L, Bech BH, Plana-Ripoll O, Bliddal M, Agerholm IE, Humaidan P, et al. Reproductive life in women with celiac disease; a nationwide, population-based matched cohort study. Hum Reprod. 2018 Jun;33(8):1538-47.

13 Singh P, Arora S, Lal S, Strand TA, Makharia GK. Celiac disease in women with infertility:
A meta-analysis. J Clin Gastroenterol. 2016 Jan;50(1):33-9.

14 Tersigni C, Castellani R, de Waure C, Fattorossi A, De Spirito M, Gasbarrini A, et al. Celiac disease and reproductive disorders: metaanalysis of epidemiologic associations and potential pathogenic mechanisms. Hum Reprod Update. 2014 Jul-Aug;20(4):582-93.

15 Khashan AS, Henriksen TB, Mortensen PB, McNamee R, McCarthy FP, Pedersen MG, et al. The impact of maternal celiac disease on birthweight and preterm birth: a Danish population-based cohort study. Hum Reprod. 2010 Feb;25(2):528-34.

16 Shah S, Leffler D. Celiac disease: an underappreciated issue in women\&\#x2019;s health [1 Engl]. Womens Health (Lond). 2010 Sep;6(5): 753-66.

17 Tiboni GM, de Vita MG, Faricelli R, Giampietro F, Liberati M. Serological testing for celiac disease in women undergoing assisted reproduction techniques. Hum Reprod. 2006 Feb;21(2):376-9.

18 van der Windt DA, Jellema P, Mulder CJ, Kneepkens CM, van der Horst HE. Diagnostic testing for celiac disease among patients with abdominal symptoms: a systematic review. JAMA. 2010 May;303(17):1738-46.

19 Secretariat MA; Medical Advisory Secretariat. Clinical utility of serologic testing for celiac disease in asymptomatic patients: an evidence-based analysis. Ont Health Technol Assess Ser. 2011;11(3):1-63.

20 Bienvenu F, Besson Duvanel C, Seignovert C, Rouzaire P, Lachaux A, Bienvenu J. Evaluation of a point-of-care test based on deamidated gliadin peptides for celiac disease screening in a large pediatric population. Eur J Gastroenterol Hepatol. 2012 Dec;24(12): 1418-23.

21 Pichler J, Zilbauer M, Torrente F, Heuschkel R, Phillips A, Salvestrini C. Feasibility of a finger prick-based self-testing kit in first- and second-degree relatives of children with coeliac disease. World J Gastroenterol. 2011 Apr; 17(14):1840-3.

22 Lau MS, Mooney PD, White WL, Rees MA, Wong SH, Hadjivassiliou M, et al. OfficeBased Point of Care Testing (IgA/IgG-Deamidated Gliadin Peptide) for Celiac Disease. Am J Gastroenterol. 2018 Aug;113(8):123846.

23 Singh P, Arora A, Strand TA, Leffler DA, Mäki M, Kelly CP, et al. Diagnostic Accuracy of Point of Care Tests for Diagnosing Celiac Disease: A Systematic Review and Meta-
Analysis. J Clin Gastroenterol. 2019 Aug; 53(7):535-42.

24 Mooney PD, Wong SH, Johnston AJ, Kurien $\mathrm{M}$, Avgerinos A, Sanders DS. Increased detection of celiac disease with measurement of deamidated gliadin peptide antibody before endoscopy. Clin Gastroenterol Hepatol. 2015 Jul;13(7):1278-1284.e1.

25 Bienvenu F, Anghel SI, Besson Duvanel C, Guillemaud J, Garnier L, Renosi F, et al. Early diagnosis of celiac disease in IgA deficient children: contribution of a point-of-care test. BMC Gastroenterol. 2014 Nov; 14:186.

26 Benkebil F, Combescure C, Anghel SI, Besson Duvanel C, Schäppi MG. Diagnostic accuracy of a new point-of-care screening assay for celiac disease. World J Gastroenterol. 2013 Aug; 19(31):5111-7.

27 Lau MS, Mooney PD, White WL, Appleby V, Moreea S, Haythem I, et al. Erratum to: Preendoscopy point of care test (Simtomax- IgA/ IgG-Deamidated Gliadin Peptide) for coeliac disease in iron deficiency anaemia: diagnostic accuracy and a cost saving economic model. BMC Gastroenterol. 2016 Oct;16(1):122.

28 Almazán MV, Ortega E, Moreno Torres R, Tovar M, Romero J, López-Casado MÁ, et al. Diagnostic screening for subclinical celiac disease using a rapid test in children aged 2-4. Pediatr Res. 2015 Sep;78(3):280-5.

29 Svedlund J, Sjödin I, Dotevall G. GSRS-a clinical rating scale for gastrointestinal symptoms in patients with irritable bowel syndrome and peptic ulcer disease. Dig Dis Sci. 1988 Feb;33(2):129-34.

30 Oberhuber G, Granditsch G, Vogelsang H. The histopathology of coeliac disease: time for a standardized report scheme for pathologists. Eur J Gastroenterol Hepatol. 1999 Oct; 11(10):1185-94.

31 Mooney PD, Hadjivassiliou M, Sanders DS. Coeliac disease. BMJ. 2014 Mar;348:g1561.

32 Ludvigsson JF, Card TR, Kaukinen K, Bai J, Zingone F, Sanders DS, et al. Screening for celiac disease in the general population and in high-risk groups. United European Gastroenterol J. 2015 Apr;3(2):106-20.

33 Fletcher RH, Fletcher SW. Clinical epidemiology. The essentials. 4th ed. Philidelphia: Lippincott Williams \& Wilkins; 2005.

34 Esteve M, Rosinach M, Llordés M, Calpe J, Montserrat G, Pujals M, et al. Case-finding in primary care for coeliac disease: accuracy and cost-effectiveness of a rapid point-of-care test. United European Gastroenterol J. 2018 Jul;6(6):855-65. 